Original Research Paper

\title{
About the Triton Structure
}

\author{
Florian Ion Tiberiu Petrescu
}

ARoTMM-IFToMM, Bucharest Polytechnic University, Bucharest, (CE), Romania

Article history

Received: 05-10-2018

Revised: $24-10-2018$

Accepted: 20-11-2018

Email: scipub02@gmail.com

\begin{abstract}
To better understand the structure of matter, it is not enough to study atoms and molecules. For living matter it is necessary to study the cell, including the mitochondrial and for matter in general, it is necessary to determine the elemental elements of the atom and further those of the nucleus, how the nucleons bind to each other, forming virtually new atomic structures if they have electrons, or new ionic structures if they suffer a lack of electrons. The present study aims to explain how the three nucleons bind to a tritium nucleus (nucleus called triton), thus forming the core of the third hydrogen isotope, a positive ion, containing two neutrons and a proton, a single particle positively charged and two neutral charged nuclear particles. For this tritium independent nucleus, called triton, the main dimensions will be determined by calculating its radius $\mathrm{R}$, which is not a constant dimension but a dynamic one, changing its magnitude in function to the velocity of the moving triton particle.
\end{abstract}

Keywords: Triton, Structure, Dimensions, Nuclear Energy

\section{Introduction}

All matter, both vivid (organic) and inorganic, is constituted at the base, from elementary particles called atoms. Atoms are formed around nuclei by capturing electrons that will rotate around nuclei in the form of electron clouds. Generally, a normal atom will contain electrons equal to the number of protons there are within its nucleus. The nucleus of the atom consists of two types of nucleons, protons (charged each with a positive charge) and neutrons (which do not have the load or otherwise have a neutral, zero charge). The nuclei are constructed starting from the minimal nucleus containing a single proton, by addition of nucleons.

If the cores could resist the electromagnetic rejection forces, they could only be made of protons. Because since the first pair of protons put together the mutual rejection forces are large enough to break the link between them, it is already necessary to connect nuclear forces (attraction) so that the core does not break. For this reason, for each added proton in the nucleus, at least one neutron should be added to contribute to the kernel balance (Halliday and Robert, 1966; Petrescu and Calautit, 2016a; 2016b; Petrescu et al., 2017a; 2017b; 2017c; 2017d, 2016a; 2016b; Petrescu, 2018; 2014; 2012a; 2012b; Petrescu and Petrescu, 2014).

For light atoms with mild nuclei (found in the first part of the diagram in Fig. 1), the required number of neutrons in the nucleus is smaller and as we move to the right towards heavier atoms and nuclei, more neutrons will be needed on the nucleus for the link between nuclear nucleons does not break. In other words, as the nucleus is larger (heavier) it will contain a larger number of neutrons within its nucleons (Halliday and Robert, 1966).

On the 45 th degree line are the nuclei that have an equal number of protons $(Z=p)$ and neutrons $(N=n)$ and above it, there are represented the heavier nuclei at which the number of neutrons in the nucleus is higher than that of the protons (Halliday and Robert, 1966). Spontaneous nuclear fission can occur only at heavy and very heavy nuclei located to the right on a larger area of the chart, while nuclear fusion is only possible at the beginning of the diagram on the left for the very first very light nuclei such as the first three isotopes of hydrogen (Kramer, 2011; Moses et al., 2009; Shultis and Faw, 2002; Krane, 1987).

The first circle drawn on the diagram in Fig. 1 corresponds to the light nucleus formed by only one neutron $(\mathrm{Z}=$ zero protons $)$ and $(\mathrm{N}=1$ neutron $)$. 


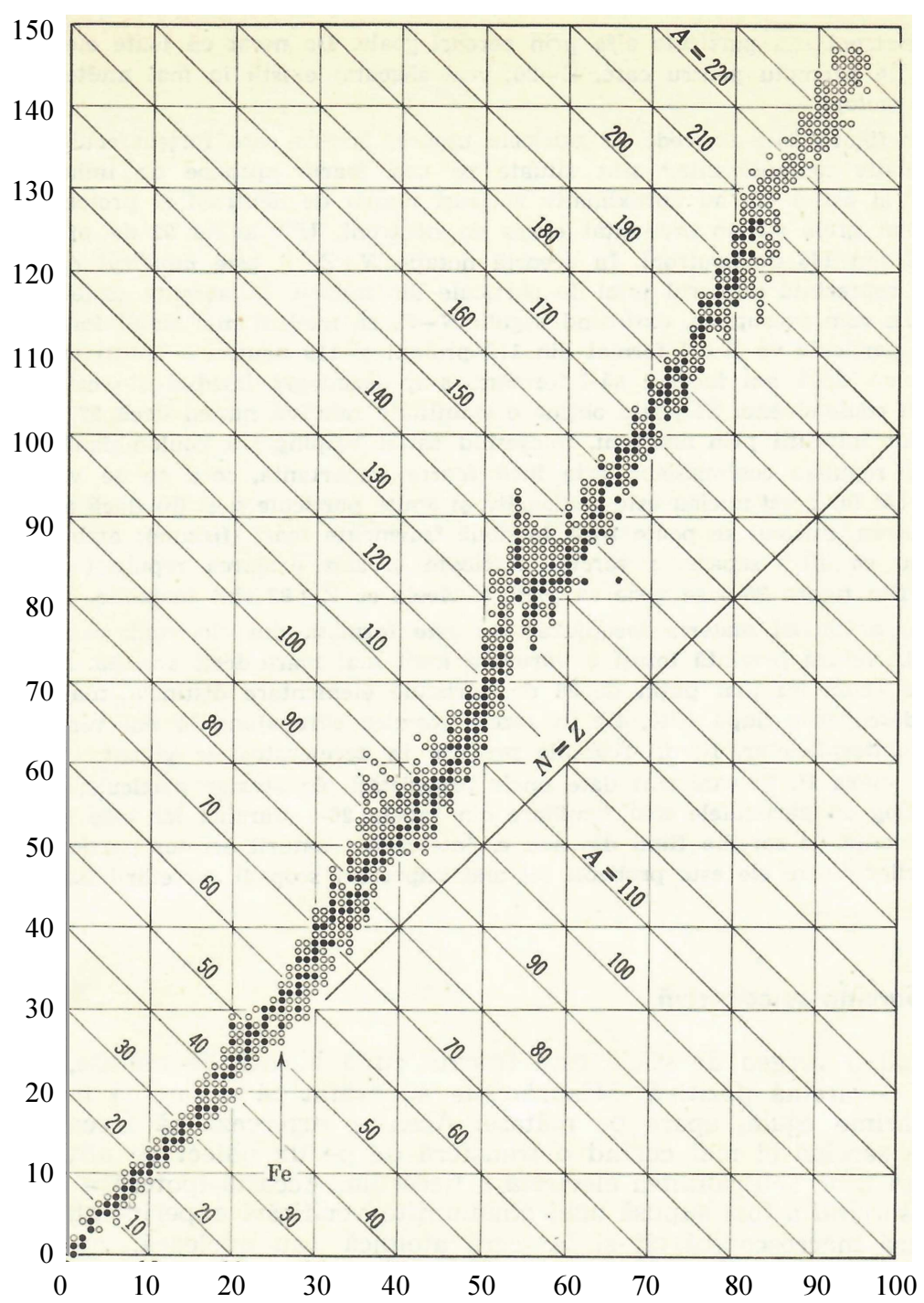

Fig. 1: Diagram of atomic cores (atomic nuclei) (Halliday and Robert, 1966)

For $Z=1$ (a single proton in the nucleus) there are three drawn variants corresponding to the three hydrogen isotopes). The zero-neutron variant $(\mathrm{N}=0)$, where the nucleus contains only one proton and will be called Proton (being the first isotope of hydrogen, which the atom is called protium and the nucleus is called proton). The second variant with a neutron $(\mathrm{N}=$ 1) in which the nucleus contains a proton and a neutron being the second hydrogen isotope (as a deuterium atom and only as the deuteron nucleus), which is located on the 45-degree line where the nuclei are balanced $(Z=N)$. And the third variant at $Z=1$ is the two neutrons $(\mathrm{N}=2)$ representing the third hydrogen isotope (as a tritium atom and as the nucleus called triton), the triton nucleus containing three nucleons, a proton and two neutrons.
In order to better understand the nuclear mechanisms represented in the diagram in Fig. 1, it should be noted that stable nuclei are represented as full circles (black), while unstable nuclei are represented as empty circles (white). So, if the proton is stable, as well as the deuteron, the triton is unstable and even more, even the neutron is now considered unstable and can decay into a proton, an electron and an antineutrino electron. Moving to $Z=2$ (two protons), we reach the helium with its three isotopes, the first two of which are stable $(\mathrm{N}=1, \mathrm{~N}=2)$ and the third is unstable $(\mathrm{N}=4)$.

\section{Materials and Methods}

As we have already shown the third hydrogen isotope, as a nucleus called triton, contains three nucleons, a proton (red) and two neutrons (blue), (Fig. 2). 


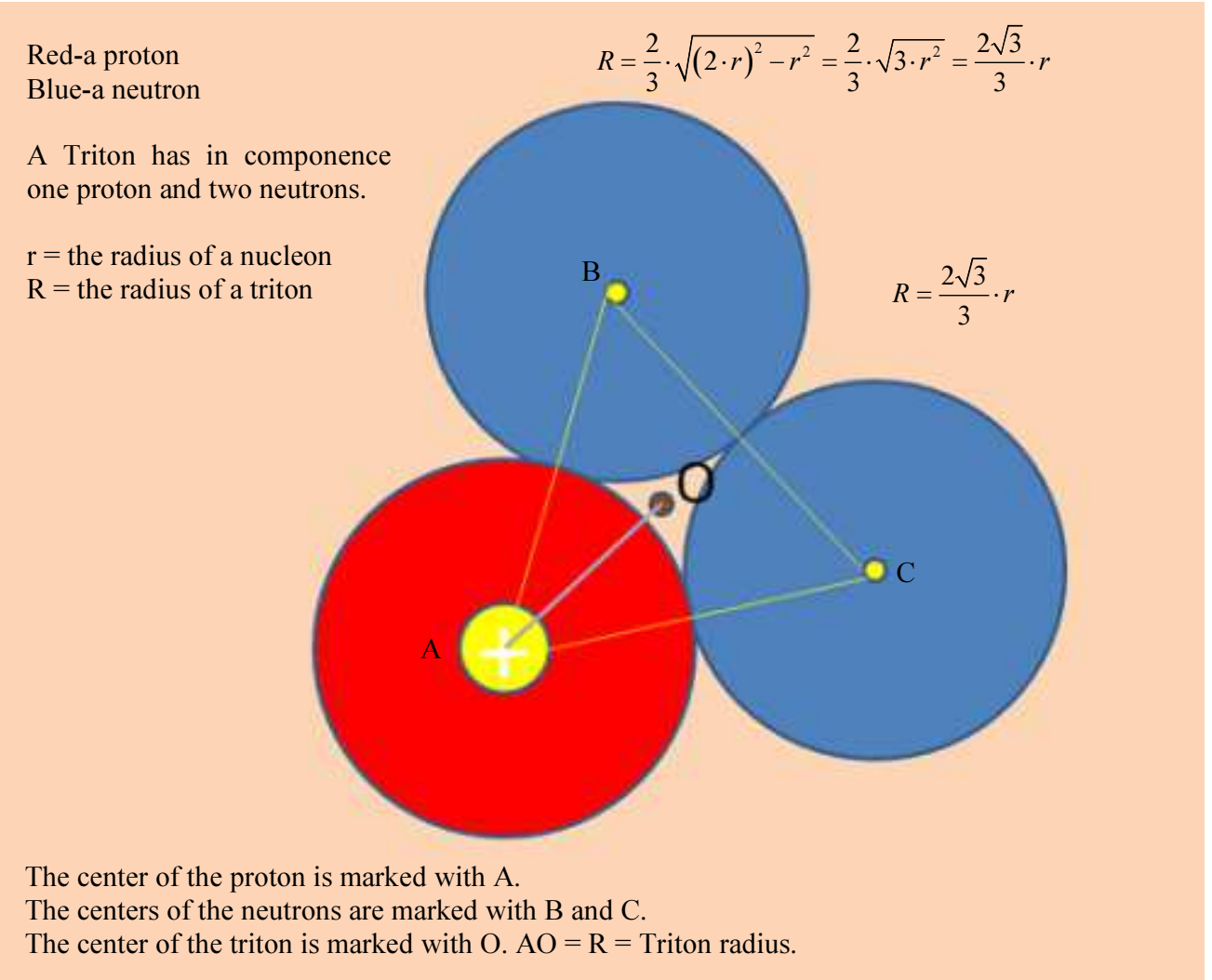

Fig. 2: The third hydrogen isotope, as a nucleus called Triton, contains three nucleons, a proton and two neutrons

The radius of the triton, $R$, is easily determined with relation 1:

$R=\frac{2}{3} \cdot \sqrt{(2 \cdot r)^{2}-r^{2}}=\frac{2}{3} \cdot \sqrt{3 \cdot r^{2}}=\frac{2 \sqrt{3}}{3} \cdot r$

The moment of mechanical inertia relative to an axis passing through the center $O$ of the system is determined by the relationship 2, where $M$ and $R$ are the mass and the radius of triton; $m$ and $r$ are the mass and the radius of one nucleon:

$$
\left\{\begin{array}{l}
J=\sum\left(\frac{2}{5} \cdot m \cdot r^{2}+m \cdot R^{2}\right)=\sum\left(\frac{2}{5} \cdot m \cdot \frac{3}{4} \cdot R^{2}+m \cdot R^{2}\right) \\
=\sum\left(\frac{3}{10} \cdot m \cdot R^{2}+m \cdot R^{2}\right)=\sum\left(\frac{13}{10} \cdot m \cdot R^{2}\right) \\
=\frac{13}{10} \cdot \sum(m) \cdot R^{2}=\frac{13}{10} \cdot M \cdot R^{2}=\frac{26}{15} \cdot M \cdot r^{2} \\
J=\frac{13}{10} \cdot M \cdot R^{2}
\end{array}\right.
$$

Any elementary moving particle possesses the kinetic energy given by the relationship 3 (composed by two components: The kinetic energy of translational motion and rotational kinetic energy of motion), where $J$ is determined with expression (2) and $M$ with relation (4) (Petrescu, 2018):

$$
E_{c}=\frac{1}{2} M \cdot v^{2}+\frac{1}{2} J \cdot \omega^{2}
$$

The mass of particle is determined with the Lorentz relationship 4 (Lorentz):

$$
M=\frac{M_{0} \cdot c}{\sqrt{c^{2}-v^{2}}}
$$

Using (2) and (4) expression (3) takes the form (5):

$E_{c}=\frac{1}{2} \frac{M_{0} \cdot c \cdot v^{2}}{\sqrt{c^{2}-v^{2}}}+\frac{1}{2} \cdot \frac{13}{10} \cdot \frac{M_{0} \cdot c \cdot R^{2}}{\sqrt{c^{2}-v^{2}}} \cdot \omega^{2}$

Pulse of the particle is written using the relation 6:

$p=M \cdot v=\frac{M_{0} \cdot c \cdot v}{\sqrt{c^{2}-v^{2}}}$

The wavelength associated with the particle can be determined with the relationship 7 (according to Louis de Broglie the pulse is conserved), where $h$ is the Planck constant:

$\lambda=\frac{h}{p}=\frac{h \cdot \sqrt{c^{2}-v^{2}}}{M_{0} \cdot c \cdot v}$ 
Wave frequency associated with the particle is determining by relationship 8 , where $c$ is the light velocity:

$$
\gamma=\frac{c}{\lambda}=\frac{c \cdot M_{0} \cdot c \cdot v}{h \cdot \sqrt{c^{2}-v^{2}}}=\frac{M_{0} \cdot c^{2} \cdot v}{h \cdot \sqrt{c^{2}-v^{2}}}
$$

The angular velocity of the particle and its square can be calculated with the relationships 9:

$$
\left\{\begin{array}{l}
\omega=2 \pi \gamma=\frac{2 \pi \cdot M_{0} \cdot c^{2} \cdot v}{h \cdot \sqrt{c^{2}-v^{2}}} \\
\omega^{2}=\frac{4 \pi^{2} \cdot M_{0}^{2} \cdot c^{4} \cdot v^{2}}{h^{2} \cdot\left(c^{2}-v^{2}\right)}
\end{array}\right.
$$

Using 9 the relationship 5 takes the forms 10:

$$
\left\{\begin{array}{l}
E_{c}=\frac{1}{2} \frac{M_{0} \cdot c \cdot v^{2}}{\sqrt{c^{2}-v^{2}}}+\frac{1}{2} \cdot \frac{13}{10} \cdot \frac{M_{0} \cdot c \cdot R^{2}}{\sqrt{c^{2}-v^{2}}} \cdot \frac{4 \pi^{2} \cdot M_{0}^{2} \cdot c^{4} \cdot v^{2}}{h^{2} \cdot\left(c^{2}-v^{2}\right)} \\
E_{c}=\frac{1}{2} \frac{M_{0} \cdot c \cdot v^{2}}{\sqrt{c^{2}-v^{2}}} \cdot\left[1+\frac{26}{5} R^{2} \cdot \pi^{2} \frac{M_{0}^{2} \cdot c^{4}}{h^{2} \cdot\left(c^{2}-v^{2}\right)}\right]
\end{array}\right.
$$

The kinetic energy of the moving particle can be determined and by the relationship 11. From the total energy of the particle in movement, subtract the total energy of the particle at rest (potential energy), (Halliday and Robert, 1966; Petrescu and Calautit, 2016a; 2016b; Petrescu, 2018):

$$
E_{c}=E-E_{0}=M \cdot c^{2}-M_{0} \cdot c^{2}=M_{0} \cdot c^{2} \cdot \frac{c-\sqrt{c^{2}-v^{2}}}{\sqrt{c^{2}-v^{2}}}
$$

\section{Results and Discussion}

Identifying the relationships 10 and 11 are obtained the expressions 12 which can determine the radius of an elementary moving particle with the particularized case when one has as particle the triton:

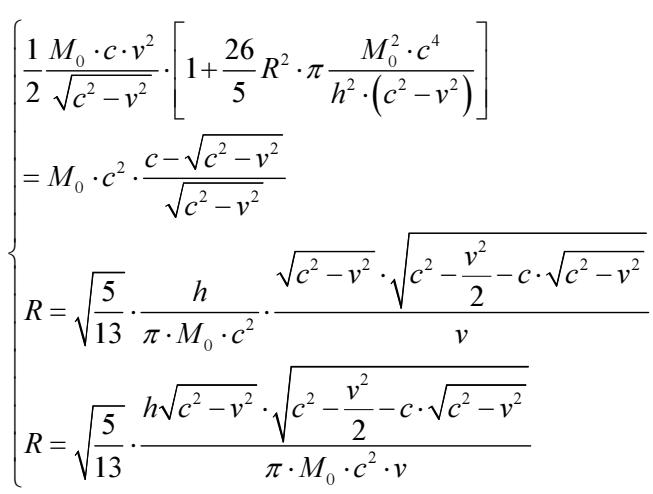

\section{Conclusion}

To better understand the structure of matter, it is not enough to study atoms and molecules. For living matter it is necessary to study the cell, including the mitochondrial and for matter in general, it is necessary to determine the elemental elements of the atom and further those of the nucleus, how the nucleons bind to each other, forming virtually new atomic structures if they have electrons, or new ionic structures if they suffer a lack of electrons. The present study aims to explain how the three nucleons bind to a tritium nucleus (nucleus called triton), thus forming the core of the third hydrogen isotope, a positive ion containing two neutrons and a proton, a single particle positively charged and two neutral charged nuclear particles. For this tritium independent nucleus, called triton, the main dimensions will be determined by calculating its radius $\mathrm{R}$, which is not a constant dimension but a dynamic one, changing its magnitude in function to the velocity of the moving triton particle.

Using the results of this original study (Equation 12), one can better understand the shape and structural dimensions of elemental particles with immediate applications in which the triton can intervene.

Obviously, the most direct applications will be used to accurately determine the dimensions of the triton depending on its velocity, velocity and kinetic energy to that the triton is accelerated to produce the fusion reaction.

\section{Acknowledgement}

This text was acknowledged and appreciated by Dr. Veturia CHIROIU Honorific member of Technical Sciences Academy of Romania (ASTR) PhD supervisor in Mechanical Engineering.

\section{Funding Information}

Research contract: Contract number 36-5-4D/1986 from 24IV1985, beneficiary CNST RO (Romanian National Center for Science and Technology) Improving dynamic mechanisms internal combustion engines. !All these Matters are Copyrighted! Copyrights:

1. New Aircraft (New Ionic or Beam Engines): no. 548 of 22-04-2010 [cgiywDssin], Aerospace Engineering

2. Some Few Specifications About the Doppler Effect to the Electromagnetic Waves: 636 of 28-05-2010 [iEtcaouxxA], physics

3. Presenting an Atomic Model and Some Possible Applications in LASER Field: nr. 639 of 29-052010 [yncngrotfo], physics

4. Some Applications in LASER Field: no. 718 of 0907-2010 [xeujouincC], physics

5. The Energies of Today and Tomorrow: nr. 819 of 30-09-2010 [kbHquxwykr], energy engineering

6. Obtaining Energy by the Annihilation of the Matter with Antimatter - The Battle for Energy: nr. 1068 of 13.03.2011 [GfEqpGDzeh], Energy Engineering. 


\section{Ethics}

This article is original and contains unpublished material. Authors declare that are not ethical issues and no conflict of interest that may arise after the publication of this manuscript.

\section{References}

Halliday, D. and R. Robert, 1966. Physics, Part II. 1st Edn., John Wiley and Sons, Inc., New York.

Kramer, D., 2011. DOE looks again at inertial fusion as a potential clean-energy source. Phys. Today, 64: 26-26. DOI: $10.1063 / 1.3563814$

Krane, K.S., 1987. Introductory Nuclear Physics. 3rd Edn., Wiley and Sons, New York, ISBN-10: 047180553X, pp: 864.

Moses, E.I., R.N. Boyd, B.A. Remington, C.J. Keane and R. Al-Ayat, 2009. The national ignition facility: Ushering in a new age for high energy density science. Phys. Plasmas, 16: 041006-041006. DOI: $10.1063 / 1.3116505$

Petrescu, F.I.T. and J.K. Calautit, 2016a. About nano fusion and dynamic fusion. Am. J. Applied Sci., 13: 261-266. DOI: 10.3844/ajassp.2016.261.266

Petrescu, F.I.T. and J.K. Calautit, 2016b. About the light dimensions. Am. J. Applied Sci., 13: 321-325. DOI: 10.3844 /ajassp.2016.321.325

Petrescu, F.I.T., 2018. About the nuclear particles' structure and dimensions. Comp. Part. Mech. DOI: $10.1007 / \mathrm{s} 40571-018-0206-7$

Petrescu, F.I.T., 2014. Nuclear Fusion. Infinite Energy, 20: 44-47.

Petrescu, F.I.T., 2012a. Cold nuclear fusion. Plasma Physics and Fusion Technology (S70), INIS.

Petrescu, F.I.T., 2012b. Cold Nuclear Fusion. Create Space Publisher, USA, ISBN-13: 978-1-4782-3426-5, pp: 80.

Petrescu, F.I.T., A. Apicella, R.V. Petrescu, S. Kozaitis and R. Bucinell et al., 2016a. Environmental protection through nuclear energy. Am. J. Applied Sci., 13: 941-946.

DOI: 10.3844 /ajassp.2016.941.946
Petrescu, R.V., R., Aversa, A., Apicella, S., Li and G., Chen et al., 2016b. Something about electron dimension. Am. J. Applied Sci., 13: 1272-1276. DOI: 10.3844/ajassp.2016.1272.1276

Petrescu, R.V., R., Aversa, S., Li, M.M., Mirsayar and R., Bucinell et al., 2017a. Electron dimensions. Am. J. Eng. Applied Sci., 10: 584-602. DOI: 10.3844/ajeassp.2017.584.602

Petrescu, R.V., R., Aversa, S., Kozaitis, A., Apicella and F.I.T., Petrescu, 2017b. Deuteron dimensions. Am. J. Eng. Applied Sci., 10: 649-654. DOI: 10.3844/ajeassp.2017.649.654

Petrescu, R.V., R., Aversa, S., Kozaitis, A., Apicella and F.I.T., Petrescu, 2017c. Some proposed solutions to achieve nuclear fusion. Am. J. Eng. Applied Sci., 10: 703-708. DOI: 10.3844/ajeassp.2017.703.708

Petrescu, R.V., R., Aversa, S., Kozaitis, A., Apicella and F.I.T., Petrescu, 2017d. Some basic reactions in nuclear fusion. Am. J. Eng. Applied Sci., 10: 709716. DOI: 10.3844 /ajeassp.2017.709.716

Petrescu, F.I.T. and R.V. Petrescu, 2014. Nuclear green energy. IJAP, 10: 3-14.

Shultis, J.K. and R.E. Faw, 2002. Fundamentals of Nuclear Science and Engineering. CRC Press, New York, ISBN-10: 0824708342, pp: 520.

\section{Nomenclature}

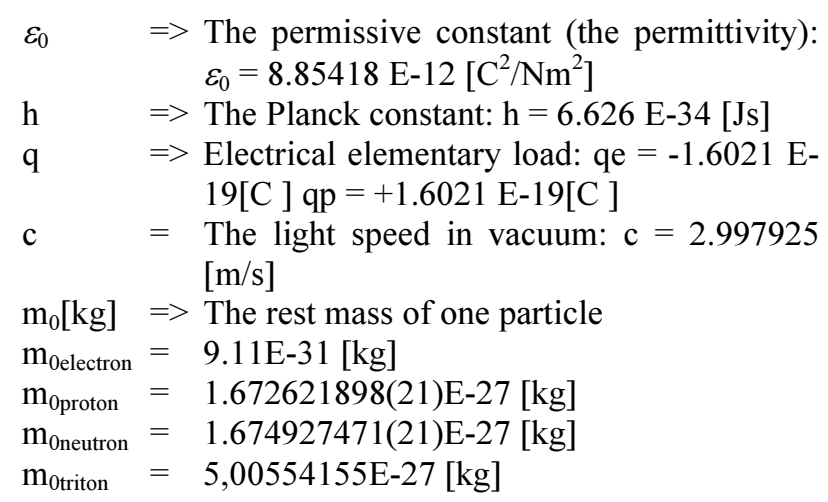

